\title{
Could the Breast Prognostic Biomarker Status Change During Disease Progression? An Immunohistochemical Comparison between Primary Tumors and Synchronous Nodal Metastasis
}

\author{
Reham Shehab El Nemr Esmail ${ }^{1 *}$, Lubna Omer El Farouk Abdel-Salam², \\ Mohammed M Abd El Ellah ${ }^{3}$
}

\begin{abstract}
Background: Prognostic biomarkers in breast cancer are routinely investigated in the primary tumors to guide further management. However, it is proposed that the expression may change during the disease progression, and may result in a different immune profile in the metastatic nodes. This work aimed to investigate the expression of breast prognostic biomarkers in primary tumors and in its axillary nodal metastasis, to estimate the possible discordant expression. Materials and Methods: 60 paired primary and axillary nodal metastasis samples were collected from patients with primary breast cancer with positive nodal deposits, diagnosed at the Maadi Military Hospital, Cairo, Egypt, during the year 2013. ER, PR and HER2 expression was assessed by immunohistochemistry in all samples Results: $48.3 \%$ of the included cases showed concordant results for both ER and PR receptors between the primary tumor and its nodal metastasis while $51.7 \%$ showed discordant results and the discordance level was statistically significant. On the other hand, $70 \%$ of the cases showed concordant Her 2 results between the primary tumors and the nodal deposits, $30 \%$ showed discordant results and the difference was significant. Conclusions: The study indicated that the discordance in ER and PR receptor expression between the primary breast tumor and their nodal metastasis may be significant. The possible switch in the biomarker status during the disease progression is worth noting and may change the patient therapeutic planning. So, whether the treatment selection should be based on biomarkers in the lymph node is a topic for further studies and future clinical trials.
\end{abstract}

Keywords: Breast cancer - axillary nodes - breast biomarkers - concordance - discordance

Asian Pac J Cancer Prev, 16 (10), 4317-4321

\section{Introduction}

Prognostic factors in breast cancer are becoming an extending list; besides the already established factors as the age, and the well-recognized and widely applied TNM staging system, other factors are being added and started to prove their significance practically. Recently, authors suggested that some non-anatomic criteria of the tumor that are not included in the TNM staging system, such as histologic grade and biomarkers, largely guide breast cancer management (Arapantoni-Dadioti et al., 2012) and (Bagaria et al., 2014).

The hormone receptor estrogen receptor (ER), progesterone receptor (PR), and epidermal growth factor receptor (Her-2/neu) status of the tumor at the initial diagnosis has now been established as a clinically important, standard-of-care parameter in treatment options selection, may reflect subsequent patient response and is thought to identify cardinal subtypes (Kinsella et al.,
2012) (Chin et al., 2014).

Thus, the introduction of hormonal therapy and selective targeted therapy, especially tamoxifen and trastuzumab, has significantly changed breast cancer outcome worldwide, and thus the current therapeutic strategies for management of cancer breast have become dependent on the accurate immunohistochemical (IHC) determination of hormone receptor status and Her2 status in order to determine the clinical utility and effectiveness of hormone-directed therapies and Herceptin (Jabbour et al., 2012) and (Patani et al., 2013)

Although they are now routinely evaluated, the endocrine markers and Her2 have proved to be potentially heterogeneous in their expression, even within the same tumor (Patani et al., 2013), the fact that raised several questions regarding the potential or possible phenotypic switch in hormonal receptors and Her-2 expression between the primary and nodal/metastatic site; whether patients should be tested for ER, PR, and Her-2/neu

${ }^{1}$ Pathology Department,National Research Center , Giza, Pathology Department, ${ }^{2}$ Kasr El Aini, Cairo University , ${ }^{3}$ Pathology Department, El Fayum University, Cairo, Egypt*For Correspondence: Rehamelnemr@Yahoo.Com 
expression in the nodal metastasis, and whether this will carry different prognostic message or not, remains elusive (Jabbour et al., 2012) (Arapantoni-Dadioti et al., 2012).

The aim of this retrospective study was to investigate the expression of breast biomarkers in primary breast cancer and in its synchronous s nodal metastases, and to estimate the percentage of cases with discordant expression.

\section{Materials and Methods}

\section{Study population}

The study population consisted of 60 paired primary and axillary nodal metastasis. Samples were collected from patients with primary breast cancer with positive nodal deposits, diagnosed at the Maadi Military Hospital, Cairo, Egypt, during the year 2013 according to the following inclusion and exclusion criteria;

The inclusion criterion was: $i$ ) Positive synchronous nodal metastasis

The exclusion criterion was: $i$ ) Cases received neo adjuvant chemo or radiotherapy.

The histopathological diagnoses with the staging were re-evaluated for confirmation (M.Mand L.O). ER, PR and HER 2 expression were assessed by immunohistochemistry (IHC) both in the primary tumors and their synchronous nodal deposits.

Immunohistochemical analysis was carried out on 4- $\mu \mathrm{m}$ sections.

All primary tumors and metastases were stained by the same person according to the same protocol to allow optimal pair-wise comparisons.

For all staining: slides were deparaffinized in xylene and rehydrated in decreasing ethanol dilutions. Endogenous peroxidase activity was blocked with $\mathrm{H} 2 \mathrm{O} 2$ in phosphate buffered saline (PBS) followed by antigen retrieval. For ER and HER2, antigen retrieval was performed in an autoclave with the slides placed in an EDTA buffer, $\mathrm{pH}=9$. For $\mathrm{PR}$ antigen retrieval was performed in citrate buffer, $\mathrm{pH}=6\left(20\right.$ minutes, $\left.100^{\circ} \mathrm{C}\right)$. A cooling off period of 30 minutes preceded the incubation ( 60 minutes, room temperature) with the primary antibodies.

Mouse monoclonal antibodies used were: ER (M7047, 1:80, DAKO, Glostrup, Denmark), PR (M3569, 1:50, DAKO) and HER2 (RM-9103-S, 1:100, Neomarkers, Lab Vision Corporation, Fremont, California, USA). For detection of the primary antibodies a poly HRP anti Mouse/Rabbit/Rat IgG (ready to use; Powervision, Immunovision Technologies, Brisbane, California, USA) was used.

Between steps, slides were washed with PBS. Finally, peroxidase activity was developed with diaminobenzidin, slides were lightly counter-stained with hematoxylin, dehydrated in increasing alcohol dilutions and cover slipped.

\section{Evaluation of IHC:}

Scoring of IHC slides was performed by two observers (R.E and L.O) in random order, blinded to other data in the paired samples. For ER and PR, the percentage of positively stained nuclei was estimated. In primary tumor samples, the adequacy of staining was checked by also evaluating the normal breast parenchyma when present (internal control).

Allred semiquantitative scoring system was used for ER and PR staining evaluation. In this system, proportion of positive cells is scored on a scale of (0-5) , where ( 0 , no staining; $1,<1 \% ; 2$, between $1 \%$ and $10 \% ; 3$, between $11 \%$ and $33 \%$; 4 , between $34 \%$ and $66 \%$ and 5 , between $67 \%$ and $100 \%$ of the cells staining)and staining intensity scored on a scale of (0-3) where (1 for weak, 2 for moderate and 3 for strong). The proportion and intensity are then summed to produce total scores from (0-8) (Brouckert et al., 2012).

HER2 expression was scored as $0,1+, 2+$ and $3+$ according to ASCO- CAP HER2 test guide line recommendations ( 2013)[7] in which , IHC 0, no staining observed or membrane staining that is incomplete and is faint/barely perceptible and within $<10 \%$ of the invasive tumor cells; IHC1+, incomplete membrane staining that is faint/barely perceptible and within $>10 \%$ of the invasive tumor cells; IHC2+, circumferential membrane staining that is incomplete and /or weak/moderate and within $>10 \%$ of the invasive tumor cells; or complete and circumferential membrane staining and within $<10 \%$ of the invasive tumor cells and IHC3+, circumferential membrane staining that is complete and intense. Cases of (IHCO) and (IHC1+) are considered negative overexpression, while cases of (IHC2+) and (IHC3+) are considered positive overexpression.

\section{Statistical methods}

Reliability of measurements was tested with two-way mixed model intra-class correlation coefficient (ICC) for average measurements agreement. The agreement between modalities was evaluated by means of ICC. (McGraw $\mathrm{KO}$, Wong SP. Forming inferences about some intra-class correlation coefficients. Psychol Methods 1996;1:30 -46.)

\section{Results} breast.

The study included 60 female patients with cancer

Table 1. Tumor Characteristics of Study Population (N=60)

\begin{tabular}{cc}
\hline & Patients, $\mathrm{n}(\%)$ \\
\hline Tumor grade & \\
1 & $0(0 \%)$ \\
2 & $42(70 \%)$ \\
3 & $18(30 \%)$ \\
Tumor stage & \\
$\leq 2 \mathrm{~cm}(\mathrm{~T} 1)$ & $6(10)$ \\
$2-5 \mathrm{~cm}(\mathrm{~T} 2)$ & $23(38.3)$ \\
$>5 \mathrm{~cm}(\mathrm{~T} 3)$ & $25(41.7)$ \\
Skin infiltration (T4) & $6(10)$ \\
Nodes & \\
$1-3(\mathrm{~N} 1)$ & $9(15)$ \\
$4-9(\mathrm{~N} 2)$ & $35(58.3)$ \\
$>9(\mathrm{~N} 3)$ & $16(26.7)$ \\
\hline
\end{tabular}


Table 2. Qualitative Study of the concordance between immunohistochemical expression of ER, PR, and Her2, in the primary breast tumor and the nodes

\begin{tabular}{|c|c|c|c|c|c|c|c|c|}
\hline & Breast & Node & $\mathrm{N}$ & $\%$ & Kappa & SE of $\mathrm{K}$ & Strength of agreement & P value \\
\hline \multirow[t]{4}{*}{ ER } & + & + & 12 & 20 & \multirow[t]{4}{*}{-0.026} & \multirow[t]{4}{*}{0.126} & \multirow[t]{4}{*}{ Worse than expected by chance } & 0.8 \\
\hline & + & - & 19 & 31.7 & & & & NS \\
\hline & - & + & 12 & 20 & & & & \\
\hline & - & - & 17 & 28.3 & & & & \\
\hline \multirow[t]{4}{*}{ PR } & + & + & 12 & 20 & \multirow[t]{4}{*}{-0.026} & \multirow[t]{4}{*}{0.126} & \multirow[t]{4}{*}{ Worse than expected by chance } & 0.8 \\
\hline & + & - & 19 & 31.7 & & & & NS \\
\hline & - & + & 12 & 20 & & & & \\
\hline & - & - & 17 & 28.3 & & & & \\
\hline \multirow[t]{4}{*}{ HER2 } & + & + & 20 & 33.3 & \multirow[t]{4}{*}{0.399} & \multirow[t]{4}{*}{0.118} & \multirow[t]{4}{*}{ Fair agreement } & 0.002 \\
\hline & + & - & 9 & 15 & & & & HS \\
\hline & - & + & 9 & 15 & & & & \\
\hline & - & - & 22 & 36.7 & & & & \\
\hline
\end{tabular}

*NB: green areas represent concordance $\%$ but yellow areas represent discordance \%, NS: non-significant, HS: highly significant.

Table 3. Quantitative Agreement (Concordance) of ER, PR and HER2 between Breast and Nodal Metastasis

\begin{tabular}{lcccc}
\hline \multicolumn{4}{c}{ Expression value } \\
ER Breast & Node & ICC & $95 \%$ CI & P value \\
& & & & NS \\
\hline PR & & & & \\
$2.23 \pm 2.30$ & $2.28 \pm 3.11$ & -0.151 & $-0.927-0.313$ & 0.704 \\
$\begin{array}{l}\text { HER2 } \\
1.18 \pm 1.32\end{array}$ & $1.35 \pm 1.30$ & 0.705 & $0.506-0.824$ & $<0.001$ \\
\hline
\end{tabular}

NB: NS: non-significant, HS: highly significant

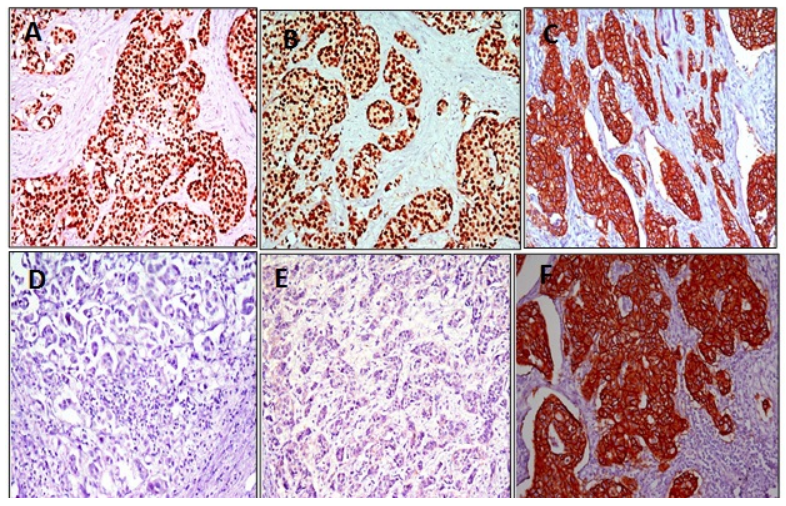

Figure 1. A case showed strong positive nuclear expression for both $E R$ and $P R$ and positive membranous overexpression of Her2 $\operatorname{scord} 3$ (a,b \& c), The same case nodal metastasis showed negative expression of ER \& PR, while the Her2

Demographic data of the patients: Age of the patients ranged from 47 up to72 years (median 56years).

Cases were divided equally between both breasts $(50 \%$ of cases were located in the right breast and the other $50 \%$ were located in the left breast).

Criteria of the tumors: $i$ ) Tumor grading and staging are listed in Table-1, ii) Four cases were multicenetric, iii) Thirty six cases showed intraductal components (ranged from minimal up to about $50 \%$ of the surface tumor area).

Results of the immunohistochemistry of the primary tumor and their synchronous nodal metastasis: (Table-2 and3) (Figures1 and 2): i) ER and PR showed nonsignificant concordance between breast andnodal

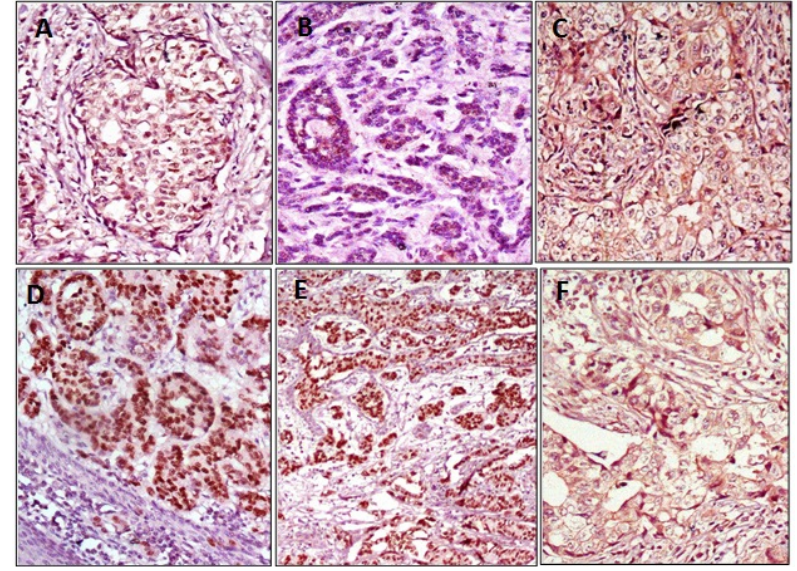

Figure 2. A case with weak ER nuclear expression, negative $P R$, and positive HER2 membranous overxpression score 2 ( $a, b, \&$ c respectively), The nodal deosit showed positive expression for both ER, PR The HER2 expression remained score2 (d, e \& frespectively)

metastasis expressions; ER and PR expressions in breast and nodes were discordant (discordance rate $51.7 \%$ for both). ii) The reverse was observed in HER 2 expressions where there was significant concordance (agreement) in the results between the primary tumor and the nodes (discordance rate 30\%). iii) There were no significant associations between the presence of concordance and tumor grade or stage ( $\mathrm{p}=0.089$ and $\mathrm{p}=0.066$ respectively). $i v)$ No significant association noted between the presence of concordance and multicentricity $\mathrm{p}=0.1$, as well as the presence of intraduct component $(\mathrm{p}=0.21$

\section{Discussion}

Estrogen receptor alpha $(\mathrm{ER} \alpha)$, progesterone receptor (PR) and human epidermal growth factor receptor 2 (HER2) have been established as principal biomarkers for breast cancer management; their evaluation has become a routine step before starting the endocrine and anti HER2 and chemotherapy (Patani et al., 2013).

The immunohistochemical expression of these markers is however heterogeneous and may differ from area to 
area within the same tumor (Thompson et al., 2010), the observation that raised the inquiry whether these biomarkers' expression could also be changeable during the disease progression and if so they may also differ in the tumor recurrences and their nodal metastasis. In this context several studies compared the ER, PR and HER2 expression both in the primary tumor and their metasynchronous metastasis. The results may largely affect the treatment plan of a metastatic tumor of breast cancer and might explain the cases of endocrine treatment and anti-Her2 failure (Aitken et al., 2010).

In the current study we aimed to investigate the degree of concordance between the immunoexpression of the breast prognostic biomarkers and their synchronous nodal metastasis. For that we compared the immunohistochemical expression of the ER, PR and Her2 in 60 retrospective primary breast cancers with their synchronous nodal metastasis.

In the current study the age ranged from 47 to 72 , with median 56. Cases were distributed equally in both breasts. The primary tumors showed various stage $(\mathrm{T}) ; 6$ cases were T1, 23 were T2, 25 were T3 and 6 cases were T4.

Four cases were multicentric and 36 cases showed intraductal components.

Immunohistochemical studies showed non-significant concordance in ER and PR expression between the tumor and their nodal metastasis (discordance rate 51.7\%). However the reverse was noted in Her2 expression; in which there was a significant concordance between the primary tumor and their nodes (discordance rate $30 \%$ ). The presence or absence of significant concordance was not associated with tumor grade, tumor stage, multicentricity or the presence of intraductal component.

The discordance between the results of ERand PR receptors was as well observed by Aitekin et al., who studied the possibility of switch in ER ,PR, andHER2 in triple negative cases, in their results they unexpectedly noticed that triple negative pattern in primary tumors are only associated with triple negative nodal disease in $76.9 \%(n=39)$ cases, while the rest of the cases showed switch in at least one of the markers, and they suggested that the latter patients might respond to hormonal therapy for which they are not currently considered (Aitekin et al., 2010). Similarly, Ba et al, noticed the discordance in ER and PR receptors in $25 \%$ and $14 \%$ of the studied cases respectively and correlated the presence of the discordance with bad prognosis. They therefore concluded that investigating the hormonal status and Her2 receptors is a promising idea to overcome therapy resistance ( $\mathrm{Ba}$ et al., 2014).

On the other hand Rokutanda et al., found that the concordance between primary tumor and their lymph node metastasis was high, however, when discordance was found in some cases it was usually linked to poor prognosis. In their results they got; almost full concordance regarding the ER status except for 1 case in which the primary tumor was negative and positive in lymph node $(\mathrm{n}=75)$. In PR discordance was $15.8 \%(\mathrm{n}=12)$, half of them changed as well from negative to positive. The discordance was $5.3 \%$ in HER $2(n=4)$, among which three had negative primaries and positive lymph nodes
(Rokutanda et al., 2012).

Similarly, Falck et al noticed that the concordance for biomarker expression in primary tumors and corresponding lymph node metastases was $93 \%$ for ER, $84 \%$ for PR, $97 \%$ for HER2. The discordant cases for HER2 status were all negative in the tumor but positive in the node (Falck et al., 2010)

Many theories tried to explain the occurrence of such switch in the hormonal and HER2 status; some authors hypothesized that the tumor cells may acquire new biological criteria to gain access to blood vessels / lymphatics, travel through circulating blood/lymph and colonize in a distant metastatic site, these molecular alterations are likely to be associated with receptor status conversion since endocrine and growth signal pathways are involved in invasion and metastasis (Maynadier et al., 2009) and (Huang et al., 2008). The conversion from positive to negative may well be explained by clonal selection of less differentiated receptor during the metastatic process (Hoefnage et al., 2010). However the conversion from negative in the primary tumor to positive in lymph nodes or metastasis is more difficult to explain, and no previous studies could provide explanation for this phenomenon, and only Hoefnagel et al., went for that false negative primary tumor results cannot be fully excluded.

The general idea of receptor conversion and subsequent molecular subtype shifting throughout the disease progression process is being gradually understood and appreciated (Salim et al., 2014); the concept was accepted and confirmed by Hoefnage et al., Lindstrom et al. and Ibrahim et al., They based their conclusions on comparing the primary tumor status with the status of their metastasis; however, these studies' results may be affected by the concept of tumor immunohistochemical heterogeneity that could be more accepted on testing core biopsies from the primary tumor-as well as the possible effect of any neoadjuvent or post-operative therapy (Hoefnage et al., 2010), (Lindstrom et al., 2012) and (Ibrahim et al., 2013).

The confirmed switch in hormonal status in this current study either in positive or negative cases may pointed to that changes in the cell biology of breast cancer would seem to occur during the disease progression.

As a conclusion: the study pointed to that the discordance in ER and PR receptor expression between the primary breast tumor and their nodal metastasis is sometimes significant. The possible switch in the biomarker status during the disease progression is worth noting, we suppose that it may be responsible for treatment resistant cases and so may change the patient therapeutic planning. So, whether treatment selection could be based on biomarkers in the lymph node, and whether this discordance may carry any prognostic message are hot topics for further studies and future clinical trials.

The authors have no conflicts of interest, and have no financial relationship with any organization.

\section{Acknowledgements}

The authors are thankful to Dr. AbdAlla Abdel Kader, head of pathology department in Maadi Military Hospital for his support and cooperation. 


\section{References}

Aitken SJ, Thomas JS, Langdon SP, et al (2010). Quantitative analysis of changes in ER, PR and HER2 expression in primary breast cancer and paired nodal metastases. Ann Oncol, 21, 1254-61.

Arapantoni-Dadioti P, Valavanis C, Gavressea T, et al. (2012). Discordant expression of hormone receptors and HER2 in breast cancer. A retrospective comparison of primary tumors with paired metachronous recurrences or metastases. $J$ BUON, 17, 277-83.

Ba JL, Liu CG, Jin F (2014). Alterations in hormonal receptor expression and HER2 status between primary breast tumors and paired nodal metastases: discordance rates and prognosis. Asian Pac J Cancer Prev, 15, 9233-9.

Bagaria SP, Ray PS, Sim MS, et al.(2014). Personalizing breast cancer staging by the inclusion of ER, PR, and HER2.JAMA Surg, 149, 125-9.

Brouckert O, Paridaens R, Floris G, et al. (2012). A critical review why assessment of steroid hormone receptors in breast cancer should be quantitative. Ann Oncol. 10, 1-7.

Chen W, Yang H, Tang WR, et al. (2014). Updated Meta-analysis on HER2 Polymorphisms and Risk of Breast Cancer: Evidence from 32 Studies. Asian Pac J Cancer Prev, 15, 9643-7.

Falck AK, Ferno M, Bendahl PO, et al. (2010). Does analysis of biomarkers in tumor cells in lymph node metastases give additional prognostic information in primary breast cancer? World J Surg, 34, 1434-41.

Hoefnagel LD, van de Vijver MJ, van Slooten HJ, et al. (2010). Receptor conversion in distant breast cancer metastases. Breast Cancer Res, 12, 75-7.

Huang TH, Wu Fand Loeb GB, et al. (2009). Upregulation of miR-21 by HER2/neu signaling promotes cell invasion. $J$ Biol Chem, 284, 18515-24.

Ibrahim T, Farolfi A, Scarpi E, et al. (2013). Hormonal receptor, human epidermal growth factor receptor-2, and Ki67 discordance between primary breast cancer and paired metastases: clinical impact. Oncology, 84, 150-7.

Jabbour MN, Massad CY, Boulos FI (2012). Variability in hormone and growth factor receptor expression in primary versus recurrent, metastatic, and post-neoadjuvant breast carcinoma. Breast Cancer Res Treat, 135, 29-37.

Kinsella MD, Nassar A, Siddiqui MT, et al. (2012). Estrogen receptor (ER), progesterone receptor (PR), and HER2 expression pre- and post- neoadjuvant chemotherapy in primary breast carcinoma: a single institutional experience. Int J Clin Exp Pathol, 5, 530-6.

Lindstrom LS, Karlsson E, Wilking UM, et al. (2012). Clinically used breast cancer markers such as estrogen receptor, progesterone receptor, and human epidermal growth factor receptor 2 are unstable throughout tumor progression. J Clin Oncol, 20, 2601-8.

Maynadier M, Nirde P, Ramirez JM, et al. (2008). Role of estrogens and their receptors in adhesion and invasiveness of breast cancer cells. Adv Exp Med Biol, 617, 485-491.

Patani N, Martin LA, Dowsett M. (2013). Biomarkers for the clinical management of breast cancer: international perspective. Int J Cancer, 133, 1-13.

Salim DK, Mutlu H, Eryilmaz MK, et al. (2014). Molecular Types and Neoadjuvant Chemotherapy in Patients with Breast Cancer- While Molecular Shifting is More Common in Luminal a Tumors, The Pathologic Complete Response is Most Frequently Observed in Her-2 Like Tumors. Asian Pac J Cancer Prev, 15, 9379-83.

Thompson A. M , Jordan LB, Quinlan P, et al. (2010). Prospective comparison of switches in biomarker status between primary and recurrent breast cancer: the Breast Recurrence In Tissues Study (BRITS). Breast Cancer Res, 12, 92-5.

Rokutanda N, Horiguchi D, Takata D,et al.(2012). Comparison of hormone receptor and HER2 expression in primary breast cancers and sentinel lymph node metastasis. Cancer Res, 72, 2-5-7.

www.cap.org/aps/docs/committees/.../summary of recommendations.pdf. 